\title{
Existe-t-il vraiment une limite génétique à la durée de la vie ?
}

L'accroissement du taux de mortalité avec l'âge est un dogme de la biologie animale. Il se résume dans la loi de Gompertz: le taux de mortalité s'élève exponentiellement avec l'âge ; dans l'espèce humaine, le temps de doublement de ce taux serait de 8 ans. Une possibilité, cependant, serait que cette loi soit en défaut aux âges très avancés. Les statistiques sur la longévité des espèces portent en effet presque toujours sur un nombre limité d'individus ; à la fin de la courbe, il ne reste qu'un très petit nombre de sujets en vie, inadapté à toute étude statistique. C'est pourquoi une équipe dirigée par Carey (Davis, CA, USA) a entrepris [1] une étude portant sur un grand nombre de mouches méditerranéennes, culminant en une expérience impliquant
1200000 mouches, réparties en groupes de 7200 , élevées au centre de Metapa, au Mexique. La courbe de mortalité obéissait d'abord aux lois classiques; mais après 30 jours la courbe s'est infléchie, et sa pente devint négative à partir de 60 jours. A cette date, il restait $0,2 \%$ du nombre initial, et l'on conçoit qu'il faille partir d'une très grande masse d'insectes pour percevoir cette inflexion. La figure 1 illustre cette courbe, comparée à celle que faisait prévoir la loi classique. Le Tableau I montre les variations de l'espérance de vie en fonction de l'âge; il indique aussi que la longévité maximale dans l'échantillon a été de 170 jours, le double de celle de 99,99\% de l'ensemble des mouches. Ce travail considérable a été effectué nécessai- rement avec des mouches génétiquement différentes, et on peut penser que certaines souches sont plus résistantes que d'autres. C'est pourquoi Curtsinger et al. (Minneapolis, MN, USA) ont examiné dix souches pures de drosophiles, en nombre naturellement plus faible (quelques milliers) [2]. Des résultats du même ordre ont été obtenus ; le taux de mortalité augmente jusqu'à 30 jours puis se stabilise ; le nombre initial d'animaux n'est pas suffisant pour qu'on puisse assister à une baisse de la courbe. Deux conclusions inattendues ressortent de ces études : (a) chez les individus les plus vieux, le taux de mortalité cesse de croître et peut même baisser ; (b) il ne semble pas y avoir de "limite génétique " à la longévité de l'espèce; certaines

Tableau I

ESPÉRANCE DE VIE EN FONCTION DE L'AGE CHEZ LES MOUCHES MÉDITERRANÉENNES (D'après [1])

\begin{tabular}{|lrcc|}
\hline $\begin{array}{c}\text { Proportion de la } \\
\text { population en vie }\end{array}$ & Nombre (arrondi) & $\begin{array}{c}\text { Age } \\
\text { (jours) }\end{array}$ & e (espérance de vie) \\
\hline 1,0 & 1200000 & 0 & 20,9 \\
0,1 & 120000 & 33 & 7,3 \\
0,01 & 12000 & 50 & 6,7 \\
0,001 & 1200 & 64 & 9,7 \\
0,0001 & 120 & 86 & 24,8 \\
0,00001 & 12 & 146 & 11,3 \\
0,000001 & 2 & 165 & 6,5 \\
\hline
\end{tabular}


mouches atteignent un âge 5 fois supérieur à celui où $90 \%$ de la population ont disparu. Reste à savoir quelles implications vont avoir ces recherches pour d'autres espèces, au premier plan la nôtre. Ces publications font en effet suite à une controverse dont la revue Science s'était fait l'écho [4]. L'allongement de la durée de vie humaine observé depuis un siècle signifie-til que la limite de la vie humaine a été fixée arbitrairement? La figure 2 montre les courbes classiques de la durée de vie depuis 1900 jusqu'à 1950 [5]. On voit que les progrès portent sur la mortalité infantile, mais aussi que la courbe descendante se rapproche d'une droite et de la verticale ; cependant toutes les

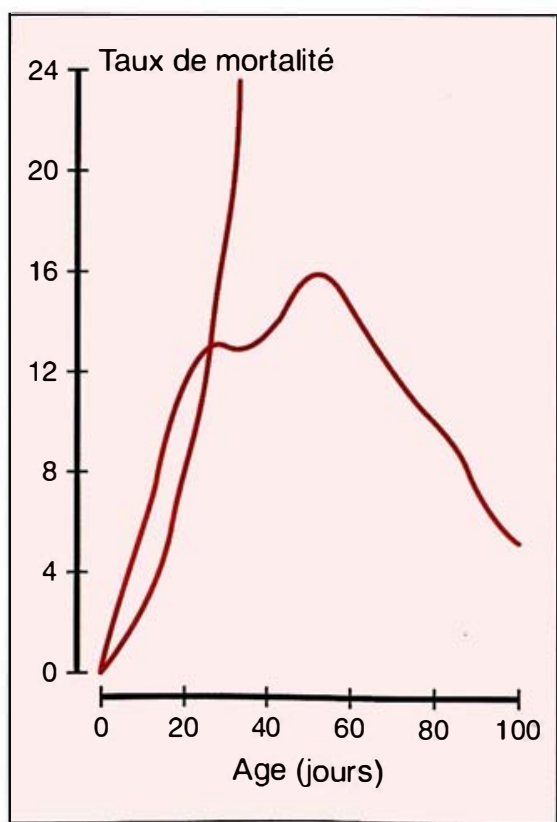

Figure 1. Comparaison entre la courbe prévue par la loi de Gompertz et celle observée par Carey et al. montrant une inflexion entre 30 et 60 jours puis une baisse de la mortalité traduite par une montée de l'espérance de vie (e). (D'après [3].)

$\mathrm{m} / \mathrm{s} n^{\circ} 10$ vol. 8 , décembre 92

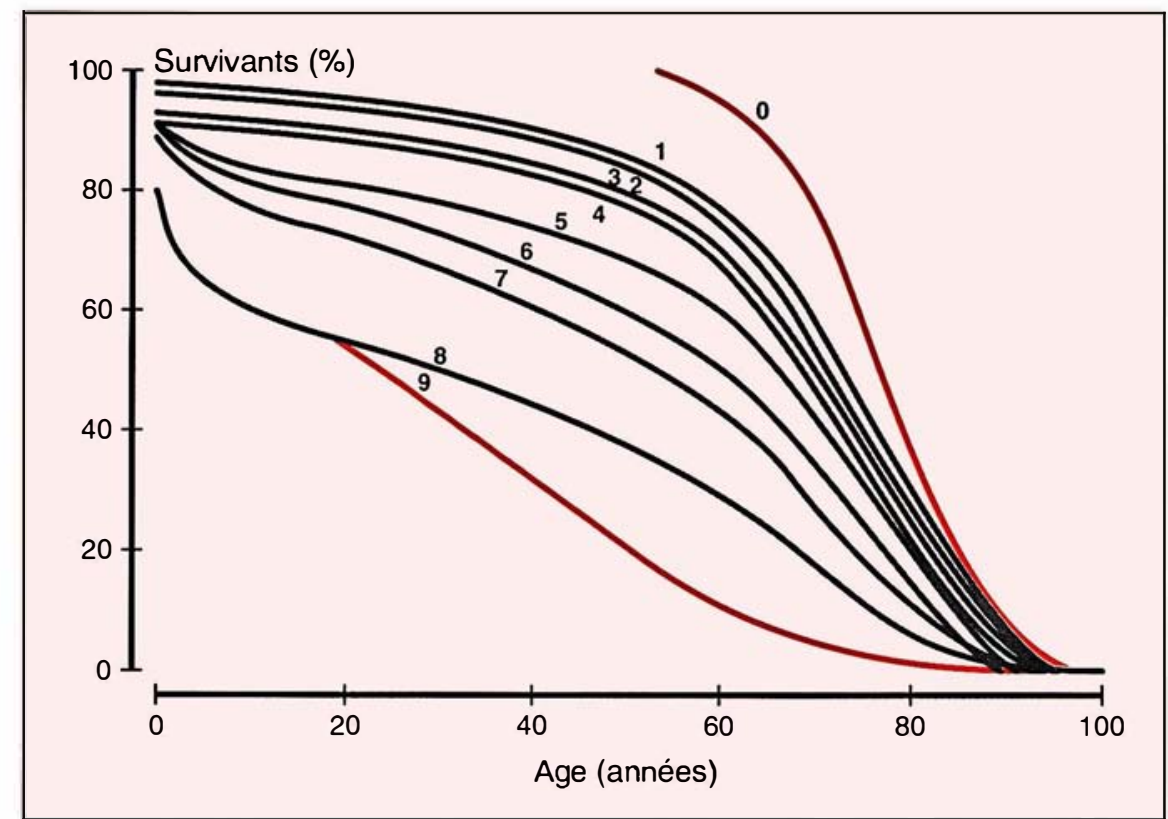

Figure 2. Courbes de mortalité de populations, entre 1900 (courbe 9) et 1940 (courbe 1). La courbe $O$ correspond à une population maintenue en bonne santé jusqu'à sa limite biologique supposée. On voit que les courbes convergent vers 90 ans mais avec une forte imprécision. (D'après [5].)

courbes semblent aboutir au même point, qui serait la limite immuable de la longévité humaine. Les résultats obtenus chez les mouches invitent à examiner de plus près la frange ultime de la courbe. J. Vaupel (Duke U et Odensee, Danemark) a émis l'hypothèse qu'à partir d'un âge à déterminer - après 85 ans, par exemple - la courbe de la vitesse de mortalité pourrait s'infléchir. Il a compulsé les registres de mortalité en Suède, et trouvé que la mortalité des Suédois (à vrai dire, surtout des Suédoises) a beaucoup diminué au cours des 50 dernières années; cette constatation, qui vaut certainement pour d'autres pays, ne permet cependant pas de prouver une élévation des potentialités vitales. L'étude des
" vieux de la vieille" (oldest old) devrait vérifier si, au milieu de la diversité génétique des humains, certains individus, épargnés par les accidents de l'existence ou plus résistants, pourraient " ne pas vieillir " ou, du moins, vieillir peu

1. Carey JR, Liebo P, Orozco D, Vaupel JW. Slowing of mortality rates at older ages in large medfly cohorts. Science $1992 ; 258$ : 457-61. 2. Curtsinger JW, Fukui HH, Townsend DR, Vaupel JW. Demography of genotypes : failure of the limited life-span paradigm in Drosophila melanogaster. Science $1992 ; 258$ : 461-3. 3. Barinaga M. Mortality : overturning received wisdom. Science $1992 ; 258$ : 398-9.

4. Barinaga M. How long is the human lifespan? Science 1991; 25 : 936-8.

5. Comfort A. The Biology of Senescence. Edimbourg: Churchill Livingstone, 1979.
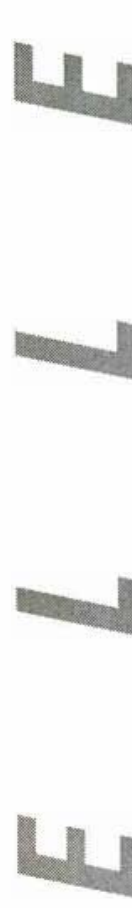

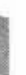

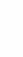

